\title{
PROSPECTS FOR JOINT GRAVITATIONAL-WAVE AND ELECTROMAGNETIC OBSERVATIONS OF NEUTRON-STAR-BLACK-HOLE COALESCING BINARIES
}

\author{
Francesco Pannarale and Frank Ohme \\ School of Physics and Astronomy, Cardiff University, The Parade, Cardiff CF24 3AA, UK; francesco.pannarale @ ligo.org, frank.ohme@ ligo.org \\ Received 2014 June 23; accepted 2014 July 10; published 2014 July 23
}

\begin{abstract}
Coalescing neutron-star-black-hole (NS-BH) binaries are a promising source of gravitational-wave (GW) signals detectable with large-scale laser interferometers such as the Advanced Laser Interferometer Gravitational-Wave Observatory and Virgo. They are also one of the main short gamma-ray burst (SGRB) progenitor candidates. If the black hole (BH) tidally disrupts its companion, an SGRB may be ignited when a sufficiently massive accretion disk forms around the remnant BH. Detecting an NS-BH coalescence both in the GW and electromagnetic (EM) spectrum offers a wealth of information about the nature of the source. How much can actually be inferred from a joint detection is unclear, however, as a mass/spin degeneracy may reduce the GW measurement accuracy. To shed light on this problem and on the potential of joint $\mathrm{EM}+\mathrm{GW}$ observations, we here combine recent semi-analytical predictions for the remnant disk mass with estimates of the parameter-space portion that is selected by a GW detection. We identify cases in which an SGRB ignition is supported, others in which it can be excluded, and finally others in which the outcome depends on the chosen model for the currently unknown NS equation of state. We pinpoint a range of systems that would allow us to place lower bounds on the equation of state stiffness if both the GW emission and its EM counterpart are observed. The methods we develop can broaden the scope of existing GW detection and parameter-estimation algorithms and could allow us to disregard about half of the templates in an NS-BH search following an SGRB trigger, increasing its speed and sensitivity.
\end{abstract}

Key words: binaries: close - equation of state - gamma-ray burst: general - gravitational waves - stars: neutron

Online-only material: color figures

\section{INTRODUCTION}

Short gamma-ray bursts (SGRBs) are brief, intense, nonrepeating flashes of radiation that, while active, outshine every other source in the gamma-ray sky. During the last decade, afterglow observations, host-galaxy identifications, and redshift measurements greatly augmented our understanding of SGRBs, but the exact nature of their progenitors is still unknown (Berger 2011). The current consensus is that the observed properties of (a subset of) SGRBs may be ascribed to matter accretion onto a stellar mass $\mathrm{BH}$ or onto a compact object that evolves into one.

The coalescence of compact binaries comprising NSs-NSBH and NS-NS systems - is therefore a natural astrophysical scenario for the production of viable SGRB progenitor candidates (Nakar 2007), as the coalescence remnant may consist of a $\mathrm{BH}$ with negligible baryon contamination along its rotation axis, surrounded by a hot, massive accretion disk (e.g., Foucart et al. 2013; Rezzolla et al. 2011) that releases energy while accreting onto the $\mathrm{BH}$.

With the imminent start of the era of second-generation laserinterferometric GW detectors, further light may be shed on compact binaries as SGRB progenitors. The upgraded detectors of the Laser Interferometer Gravitational-Wave Observatory (LIGO; Harry \& The LIGO Scientific Collaboration 2010) and Virgo (Virgo Collaboration 2009) collaborations, and the newly built Kamioka Gravitational wave detector (KAGRA; Somiya 2012) will allow for directly observing the last instants of the evolution of NS systems via the detection of their GW emission, providing unprecedented information about the physical nature of these systems.

In this Letter, we focus on NS-BH systems, noting that a $\mathrm{GW}$ observation of these binaries would constitute their first direct detection, as opposed to already observable NS-NS systems (Kiziltan et al. 2013). At its design sensitivity ( 2019 ; LIGO Scientific Collaboration et al. 2013), Advanced LIGO is expected to detect 0.03-4.4 NS-BH mergers per year in single detector mode, while the expected detection rate for a network of three detectors operating at this sensitivity is $0.07-9.4$ events per year (Dominik et al. 2014).

Within the GW community, several NS-BH binary studies focused on the signatures left by the currently unknown NS equation of state (EOS; Lattimer \& Prakash 2007) on the emitted GW signal and on the possibility of constraining the equation of state (EOS) (e.g., Kyutoku et al. 2010; Pannarale et al. 2011, 2013; Lackey et al. 2012, 2014; Wade et al. 2014). Our analysis considers only the inspiral portion of the GW signal, since a point-particle description of this signal is accurate enough for detection purposes, as shown by Pannarale et al. (2011). However, we go beyond the GW detection scenario and examine the case of joint (inspiral) GW-EM detections. We show where in the NS-BH parameter space such detections are possible and address the idea of using them to constrain the NS EOS. Additionally, we discuss that the framework we develop would allow us to (1) assess the importance of an EM follow-up to a GW detection, and (2) to improve the performance of offline GW searches following SGRB triggers.

Recently, Maselli \& Ferrari (2014) addressed similar issues, but with a different scope. Assuming GW measurements of the parameters of non-precessing binaries, including the NS tidal deformability, they estimated the accuracy with which these parameters are determined by a linear order Fisher-matrix approximation. Consistently with this approximation, they used a Gaussian probability distribution to infer the chances of finding a coincident EM merger signature. We go beyond this study by identifying parameter degeneracies in the GW measurement through a principal component analysis (PCA): this enables us 
to present a complete analysis of the relevant NS-BH parameter space without relying on a few cases and high signal-to-noise ratios $(\mathrm{S} / \mathrm{Ns})$. Additionally, we ensure that all our estimates are conservative, particularly when addressing precessing $\mathrm{BH}$ spins, so that we identify parameter-space regions where the mere existence of a coincident GW-EM detection allows us to put lower bounds on the NS EOS stiffness, without directly measuring any tidal deformability and even in the presence of large uncertainties. Conversely, we identify regions where the merger dynamics does not support any EM emission. Relating this parameter-space portion to the regions covered by alignedspin templates, we suggest removing templates with no potential EM-counterpart to optimize SGRB-targeted GW searches.

\section{METHODOLOGY}

\subsection{Gravitational-wave Measurement}

The most sensitive method to detect GWs of known signature in noise-dominated instrument data is to cross correlate the data with theoretically predicted waveforms. This relies on accurate descriptions of the expected signals. We model compact binary inspiral waveforms by a standard frequency-domain post-Newtonian (PN) approximant (Damour et al. 2001) in the form used by Ohme et al. (2013). Even if the model were to perfectly describe the expected signal, which we assume here for simplicity, the accuracy of inferred binary parameters is limited by the fact that differences between signals from distinct sources may be undetectable below the inevitable noise floor. Thus, GW measurements only select a parameter-space region consistent with an observation; we shall summarize the method we employ to identify this region below. Note that we refer to the fiducial signal as "target signal," whereas "templates" denote waveform models used to analyze the data.

Various approaches estimate the accuracy of GW measurements, ranging from linear-order approximations (see Vallisneri 2008 for details on the Fisher-information-matrix approach) to multi-dimensional numerical analysis techniques (see Aasi et al. 2013 for recent results). In this Letter, we identify parameter degeneracies through a semi-analytical PCA, as outlined in Ohme et al. (2013). This method was shown to be more accurate than simple linear-order approximations, and was successfully implemented in GW search algorithms as an integral part of the template bank construction (Harry et al. 2014). While only the above mentioned numerical techniques take advantage of the full detector data information, our PCA is sufficiently accurate to demonstrate the general idea presented in this Letter and simultaneously allows us to analyze a large parameter space.

The details of the algorithm are as follows. We consider an NS-BH GW source and parameterize it by the constituent masses $M_{\mathrm{BH}}$ and $M_{\mathrm{NS}}$ and the $\mathrm{BH}$ spin. We neglect the NS spin as it is expected to be small in compact binaries (Bildsten \& Cutler 1992; Kochanek 1992). For templates, we also exclude precession by taking the $\mathrm{BH}$ spin to be aligned with the total orbital angular momentum, $\hat{L}$, with a dimensionless spin projection $\chi_{\hat{L}, \mathrm{BH}} \in[-1,1]$. This restriction will be relaxed for target signals as discussed in the following section. As all other source parameters, such as distance, sky location, polarization, hardly correlate with the parameters we consider, we leave them to dedicated studies (e.g., Singer et al. 2014).

We then perform a PCA with the frequency-domain PN waveform model, as implemented by Ohme et al. (2013), assuming the Advanced LIGO zero-detuned, high-power mode design sensitivity (Shoemaker 2010), with $15 \mathrm{~Hz}$ lower cutoff. The high-frequency cutoff is given by the frequency of the last stable circular orbit around a Schwarzschild BH with mass $M_{\mathrm{BH}}+M_{\mathrm{NS}}$. The PCA provides the parameter-space directions that are well constrained by $\mathrm{GW}$ observations. We take the first two of these principal components and assume them to be measured exactly at the values of our fiducial target system, which reduces the three-dimensional parameter space $\left(M_{\mathrm{NS}}, M_{\mathrm{BH}}, \chi_{\hat{L}, \mathrm{BH}}\right)$ to a one-dimensional (1D) line consistent with the target.

The result we obtain is interpreted as follows. Given a GW detection, we can measure two values very accurately. To a good approximation, the first one is described by the chirp mass:

$$
M_{\text {Chirp }}=\frac{\left(M_{\mathrm{NS}} M_{\mathrm{BH}}\right)^{3 / 5}}{\left(M_{\mathrm{NS}}+M_{\mathrm{BH}}\right)^{1 / 5}},
$$

whereas the second one restricts the range of consistent parameters to a narrow line in the mass-ratio/spin space (at constant $M_{\text {Chirp }}$ ).

We neglect $\mathrm{S} / \mathrm{N}$-dependent uncertainties on these two bestmeasured parameter combinations, as they are too small to affect our analysis. Specifically, all GW-degenerate lines we shall show below are clearly distinguishable at any $\mathrm{S} / \mathrm{N}$ sufficient for detection (see Section III in Ohme et al. 2013 for details). Incorporating third principal component information would restrict the 1D line to a finite length, but we make the conservative assumption that this would not be more restrictive than the physical bounds imposed by cosmic censorship $\left(\left|\chi_{\mathrm{BH}}\right| \leqslant 1\right)$ and the plausible NS mass range. As a technical caveat, note that although the functional form of the principal components only weakly depends on the parameter-space point one is considering, a total-mass dependence is inherited from the upper cutoff frequency: we take care of this by re-calculating the principal components for a range of target sources.

\subsection{Electromagnetic Counterpart}

We use the baryonic mass $M_{\text {disk }}$ of the disk remnant of NS-BH binaries as a proxy for EM counterparts: if $M_{\text {disk }}$ exceeds a threshold mass $M_{\text {Threshold }}$, then the merger can produce a counterpart, albeit not necessarily a detectable one, otherwise it is treated as electromagnetically silent. We determine $M_{\text {disk }}$ through the fit of Foucart (2012) to numerical-relativity diskmass predictions for aligned NS-BH mergers (Equations (7), (8), (12), and (13) therein). For misaligned mergers, we generalize this formula as suggested by Stone et al. (2013; see the text supporting Equation (9) therein). While our approach relies on this fit, any alternative and possibly improved method could be used in future applications. Unless otherwise noted, we set $M_{\text {Threshold }}=0.03 M_{\odot}:$ this is on the low end of the disk-mass values expected to allow for SGRBs $1 \mathrm{~s}$ in duration (e.g., Stone et al. 2013, end of Section III).

The following procedure combines EM prospects with GW measurements. For each fiducial target signal, we calculate $M_{\text {disk }}$ for all sources selected by a GW measurement (see Section 2.1). In addition to the parameters $M_{\mathrm{NS}}, M_{\mathrm{BH}}$, and $\chi_{\hat{L}, \mathrm{BH}}$, we consider various NS EOSs and allow the $\mathrm{BH}$ spin of the target signal to be tilted with respect to $\hat{L}$. We make the conservative assumption that precessing systems, if detected, are identified by the nonprecessing template that correctly captures the secular inspiral rate. Following Schmidt et al. (2012) and Pekowsky et al. (2013), we define this template by the same mass parameters as the signal and the projection of the generic spin onto $\hat{L}$. 


$$
M_{\mathrm{NS}}=1.35 M_{\odot} \text { and } \chi_{\hat{L}, \mathrm{BH}}=0.33
$$

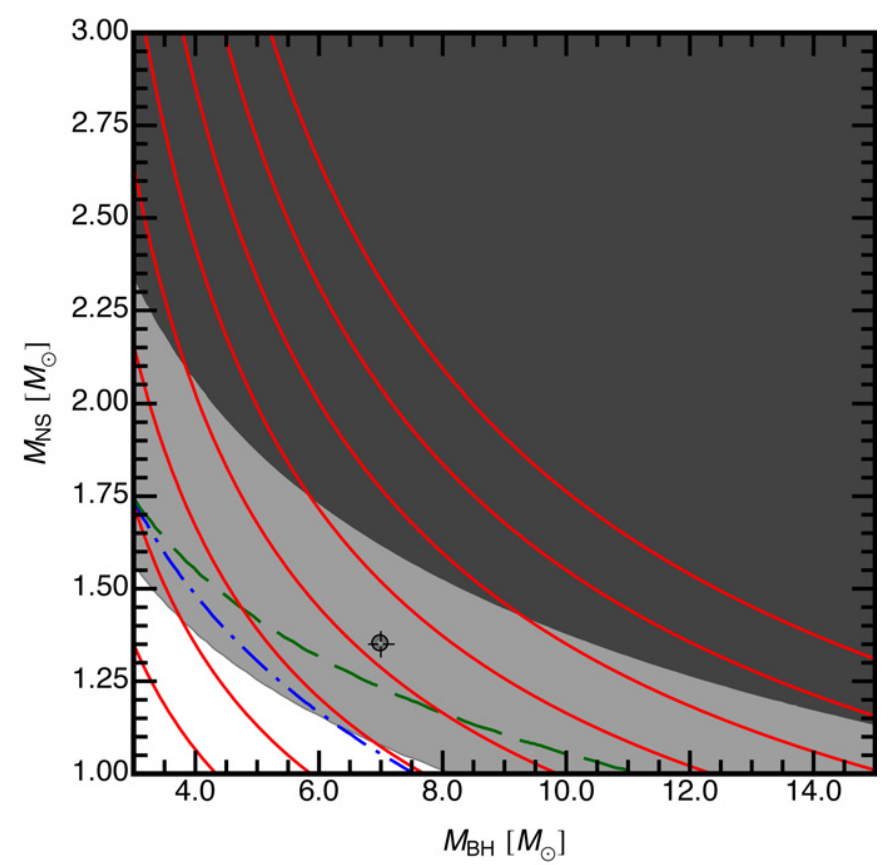

$$
M_{\mathrm{NS}}=1.35 M_{\odot} \text { and } \chi_{\hat{L}, \mathrm{BH}}=0.66
$$

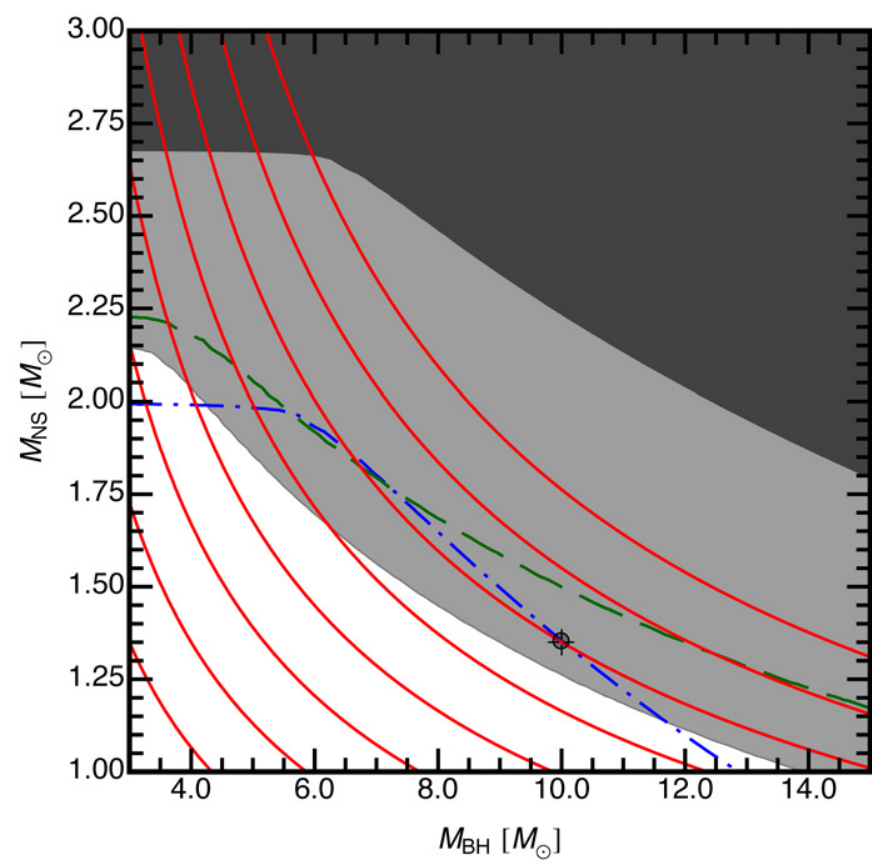

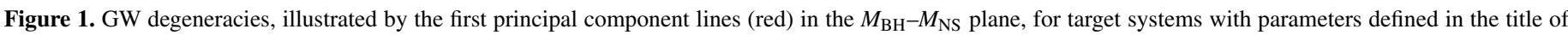

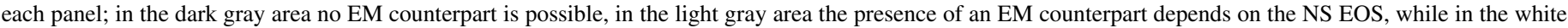

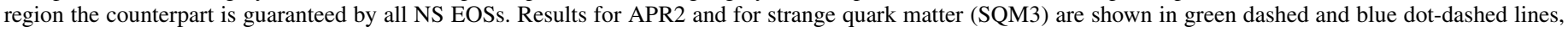
respectively.

(A color version of this figure is available in the online journal.)

We will quote results for the WFF1 (Wiringa et al. 1988), PS (Pandharipande \& Smith 1975), APR2 (Akmal \& Pandharipande 1997; Akmal et al. 1998), and SQM3 (Witten 1984; Farhi \& Jaffe 1984; Alcock \& Olinto 1988) EOSs. Although superseded, the first two EOSs yield extremely compact and extremely large NSs, respectively, generating results that bracket the ones produced by all other EOSs. APR2, on the other hand, is the most complete nuclear many-body EOS to date and is supported by current nuclear physics and astrophysical constraints: it may be thought of as a reference for our NS EOS best guess. Finally, SQM3 is an exotic EOSs employed to illustrate the case of strange quark stars; however, no strangestar-BH merger numerical simulations are available, so this is untested territory for the $M_{\text {disk }}$ fit.

Given an EOS, the techniques just described allow us to (1) split the parameter space into an EM-silent region (where $M_{\text {disk }}<M_{\text {Threshold }}$ ) and an EM-loud one (where $M_{\text {disk }}>$ $M_{\text {Threshold }}$ ), and to (2) study GW degeneracies throughout the parameter space.

\section{RESULTS}

Given a set of NS-BH parameters, as the NS EOS is softened and the NS radius decreases, $M_{\text {disk }}$ decreases due to the increased difficulty in tidally disrupting the NS. The volume of the (potentially) EM-loud parameter-space region decreases consequently. Similarly, the stiffer the EOS, the higher the chances of having an EM counterpart (Pannarale et al. 2011).

A second, less intuitive aspect is related to the $\mathrm{BH}$ spin. All "GW parameters" $\left(M_{\mathrm{NS}}, M_{\mathrm{BH}}, \chi_{\hat{L}, \mathrm{BH}}\right)$ being fixed, a higher BH spin magnitude yields a higher $M_{\text {disk }}$, because increasing $\chi_{\mathrm{BH}}$ shrinks the innermost stable orbit more rapidly than the tidal disruption orbit: the greater the difference between the radii of these two orbits, the higher $M_{\text {disk }}$. We remark that this statement on the dependency of $M_{\text {disk }}$ on $\chi_{\mathrm{BH}}$ is more general than ones for aligned mergers, as we allow for the $\mathrm{BH}$ spin to be tilted with respect to $\hat{L}$. While we assume that systems with equal values of $M_{\mathrm{NS}}, M_{\mathrm{BH}}$, and $\chi_{\hat{L}, \mathrm{BH}}$ emit similar $\mathrm{GW}$ signals, the chances of having EM counterparts depend on $\chi_{\mathrm{BH}}$ : setting $\chi_{\mathrm{BH}}=0.998$ (Thorne 1974) in our analysis therefore allows us to make conservative statements about the EM counterpart. In other words, if $M_{\text {disk }}<M_{\text {Threshold }}$ for $\chi_{\mathrm{BH}}=0.998$ and a given set of $M_{\mathrm{NS}}, M_{\mathrm{BH}}$, and $\chi_{\hat{L} \text {, BH }}$ values, no EM emission is expected to be associated with NS-BH mergers with the same mass and aligned-spin component values. Bearing this in mind, from here onward we will consider $\chi_{\mathrm{BH}}=0.998$ only.

Figures 1 and 2 are representative of our results. In Figure 1, we pick a family of target systems with $M_{\mathrm{NS}}=1.35 M_{\odot}$ and $\chi_{\hat{L}, \mathrm{BH}}=0.33(0.66)$ in the left (right) panel. We then span the template $M_{\mathrm{BH}}-M_{\mathrm{NS}}$ plane and illustrate the regions selected by GW measurements as red solid curves. These are lines of constant first principal component, essentially constant $M_{\text {Chirp }}$, along which the template $\chi_{\hat{L}, \mathrm{BH}}$ varies. Along a given curve, a lower (higher) template $M_{\mathrm{BH}}$ requires a higher (lower) $M_{\mathrm{NS}}$ to preserve $M_{\text {Chirp }}$, and a lower (higher) $\chi_{\hat{L}, \mathrm{BH}}$ to ensure a high match between the template and the target that lives on that same curve.

The dark area in the background indicates that no EM counterpart is available, because $M_{\text {disk }}<M_{\text {Threshold }}$ for any EOS. On the boundary between the two gray areas, $M_{\text {disk }}=$ $M_{\text {Threshold }}$ for the PS EOS: any NS-BH binary below it is expected to have an EM counterpart if we assume the PS EOS to be valid. Notice that the maximum NS mass can also contribute to shaping this curve: this is the case of the flattening at $M_{\mathrm{NS}} \simeq 2.66 M_{\odot}$ in the right panel.

On the boundary between the light gray and the white areas $M_{\text {disk }}=M_{\text {Threshold }}$ for the WFF1 EOS. Therefore, in 

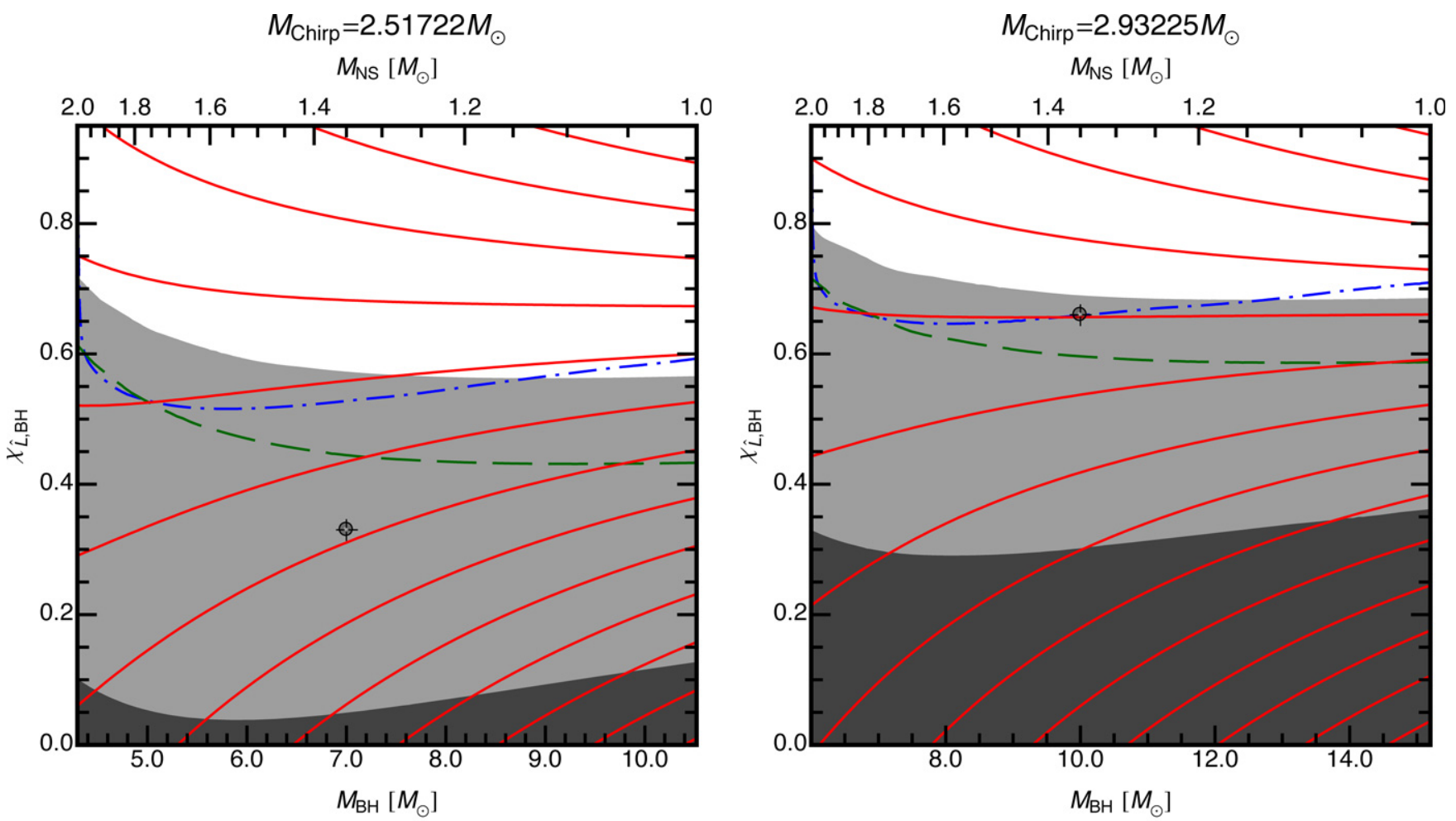

Figure 2. Second principal component lines in the $M_{\mathrm{BH}}-\chi_{\hat{L}, \mathrm{BH}}$ plane for constant $M_{\mathrm{Chirp}}$, as defined in the title of each panel (see Figure 1 for the color code). (A color version of this figure is available in the online journal.)

the light gray region, $M_{\text {disk }}$ can exceed $M_{\text {Threshold }}$, depending on the NS EOS. Within this region, we explicitly show the $M_{\text {disk }}=M_{\text {Threshold }}$ curve for the "standard" APR2 EOS in dashed green.

The white region denotes NS-BH binaries with $M_{\text {disk }}>$ $M_{\text {Threshold }}$ for any EOS. Finally, the dot-dashed blue line marks the $M_{\text {disk }}=M_{\text {Threshold }}$ boundary for strange quark stars (SQM3 EOS). Once more, the maximum mass supported by the EOS flattens the curve for moderate-to-high $\chi_{\hat{L}, \mathrm{BH}}$ values.

Figure 2 uses the same color code. Here we consider a fixed $M_{\text {Chirp }}$ target-system family and show the behavior of the second GW principal component in the template $M_{\mathrm{BH}}-\chi_{\hat{L}, \mathrm{BH}}$ plane. The $M_{\text {Chirp }}$ value corresponds to a $1.35 M_{\odot}+7(10) M_{\odot}$ system in the left(right) panel. On a given side of Figures 1 and 2, crosshairs denote the one target binary with $M_{\mathrm{NS}}, \chi_{\hat{L} \text {, BH }}$, and $M_{\text {Chirp }}$ as specified by the two panel titles on that side. This allows us to visually follow a distinct binary when varying $\chi_{\hat{L}, \mathrm{BH}}$ and $M_{\text {Chirp }}$.

A number of observations can be made. There are parameterspace regions in which joint GW-EM detections are clearly possible (white), others in which they are not possible (dark gray), and finally ones in which this possibility is EOS dependent. Quantitative information of this kind would be valuable when deciding whether to send an EM alert following an NS-BH GW detection, or when performing a GW follow-up to an SGRB trigger (see next section).

We find that increasing (decreasing) the target $\chi_{\hat{L}, \mathrm{BH}}\left(M_{\text {Chirp }}\right)$ enhances the chances of having an EM counterpart, as a larger portion of the parameter-space slice is EM-active. This generalizes the well-established behavior of $M_{\text {disk }}$ (i.e., it grows with the BH spin magnitude in aligned-spin binaries, but decreases for increasing $M_{\mathrm{BH}}$ ) and translates it into terms relevant to GW science.

Interestingly, just a few principal component lines cut through the entire EOS-sensitive region. Joint GW-EM detections can thus constrain the NS EOS, despite the GW measurement degeneracies. More specifically, a joint detection could potentially place a lower bound on the EOS stiffness. If an NS-BH GW signal is detected, the source $M_{\text {Chirp }}$ and second principal component can be determined. The presence of, say, an SGRB counterpart would indicate that part of the NS matter survived the merger, thus reducing the size of the light gray area in our Figure 1 and 2 examples. This effectively places a lower limit on the NS compactness and hence on the EOS stiffness.

A direct consequence of the previous point is that a single joint detection could exclude the presence of a strange star in the progenitor. As an example, if the principal component values of the target we fixed in the left panels of Figures 1 and 2 are measured, and an SGRB signal is detected, this could not be supported by the SQM3 EOS.

\section{DISCUSSION}

In this Letter, we considered the GW and EM emission of NS-BH binaries. We used PCA techniques for the GW inspiral signal and a proxy to determine the presence/absence of an EM counterpart. We explained how joint NS-BH binary merger detections can place lower limits on the NS EOS stiffness that constrain the EOS, potentially excluding strange quark matter EOSs with a single joint detection. Since our statements are mainly based on semi-analytical expressions derived from either numerical simulations or a PCA, this analysis can be performed quickly, making it an ideal addition to existing $\mathrm{GW}$ search and parameter-estimation pipelines.

Our method may also be used as a framework to assess the importance of sending alerts for EM follow-ups to NS-BH GW detections. Conversely, a speed-up of offline NS-BH GW searches in coincidence with SGRB triggers is possible: the number of templates used in the search may be reduced by 


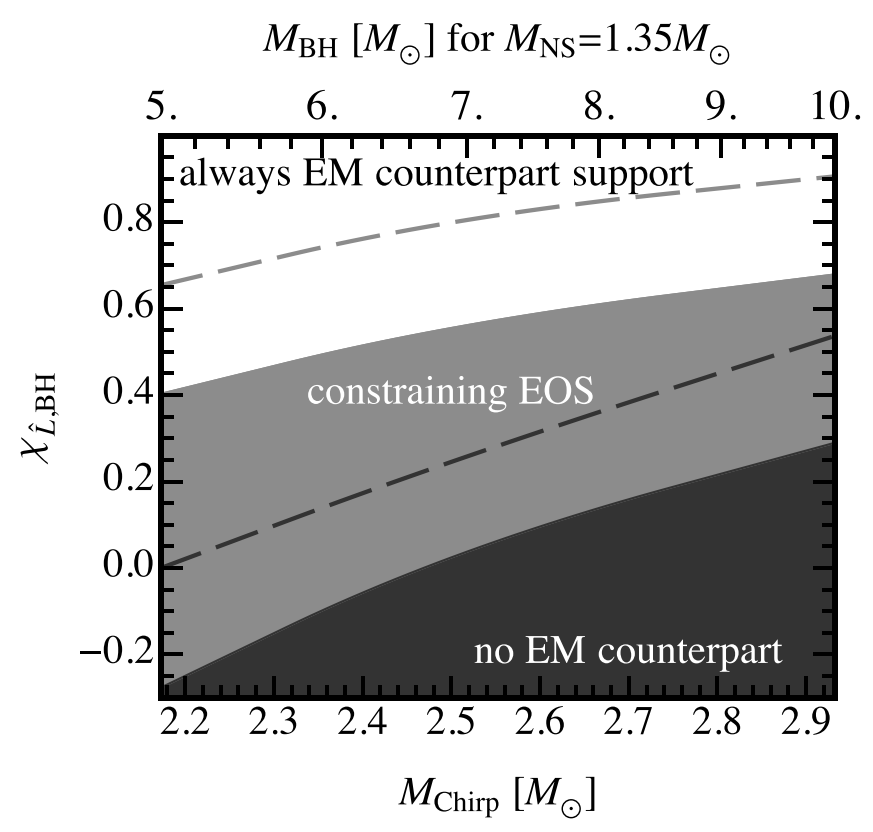

Figure 3. $M_{\text {Chirp }}-\chi_{\hat{L}, \mathrm{BH}}$ combinations for which an EM counterpart is not supported (dark gray), EM counterpart support is EOS dependent (light gray), and no EOS supports an EM counterpart (white) for $\chi_{\mathrm{BH}}=0.998$. Dashed lines denote the boundaries between the regions for aligned-spin binaries.

identifying NS-BH systems that no EOS supports as SGRB progenitors. This would also improve the search sensitivity, as keeping templates that cannot be associated with an SGRB may harm the search by increasing the false-alarm rate, which only higher $\mathrm{S} / \mathrm{N}$ thresholds could counteract.

Figure 3 illustrates the parameter-space regions where the above mentioned applications are potentially useful. In the $M_{\text {Chirp }}-\chi_{\hat{L}, \text { BH }}$ plane-the relevant physical quantities picked up by the $\mathrm{GW}$ PCA-we indicate for $\chi_{\mathrm{BH}}=0.998$ where EM counterparts are supported (white), where they are unlikely (dark gray), and where their presence allows for constraining the EOS. For reference, we also include the region boundaries for $\chi_{\mathrm{BH}} \equiv \chi_{\hat{L}, \mathrm{BH}}$ as dashed lines. Given an $M_{\text {Chirp }}$, the individual masses determine the $\chi_{\hat{L} \text {, BH }}$ required to be in one of those regions (Figure 2): we plot the minimal $\chi_{\hat{L}, \mathrm{BH}}$ that allows for constraining the EOS (lower contour) and the minimal $\chi_{\hat{L}, \mathrm{BH}}$ that ensures that $M_{\text {disk }}>M_{\text {Threshold }}$ (upper contour).

Throughout this Letter, we made very conservative assumptions about our ability to measure parameters via GW observations, and we chose an almost extremal $\chi_{\mathrm{BH}}$ for a given $\chi_{\hat{L}, \mathrm{BH}}$, favoring the onset of SGRBs. Consequently, we can conservatively estimate the volume of parameter space where an EM counterpart cannot be ignited. Using a $2 \mathrm{H}$ two-piecewisepolytrope EOS (Kyutoku et al. 2010), which gives a high maximum $M_{\mathrm{NS}}$ of $\sim 2.8 M_{\odot}$ and favors high $M_{\text {disk }}$ values by yielding large NS radii, we span the intervals $M_{\mathrm{NS}} \in[1,2.8] M_{\odot}$, $M_{\mathrm{BH}} \in[3,15] M_{\odot}$, and $\chi_{\hat{L}, \mathrm{BH}} \in[-0.95,0.95]$, keeping $\chi_{\mathrm{BH}}=0.998$. We find that $M_{\text {disk }}=0 M_{\odot}$ in $\sim 65 \%$ of the volume of this parameter space, indicating that at most $\sim 35 \%$ of the parameter space is useful for SGRB trigger follow-ups. This reduces to $\sim 25 \%$ when considering aligned-spin cases only, i.e., when $\chi_{\mathrm{BH}} \equiv \chi_{\hat{L}, \mathrm{BH}}$.

This has practical consequences for targeted $\mathrm{GW}$ searches following up EM detections. Assuming an aligned-spin templatebased search, as advised by Dal Canton et al. (2014), each tem- plate covers a section of the parameter space, including precessing binaries. We can now conservatively determine whether this section could produce an EM signature: combining our results with the proper template-bank density (Harry et al. 2014), we find that $43 \%$ of the templates in the above mentioned parameter space cover a region with vanishing $M_{\text {disk }}$. For non-precessing signals, this increases to $48 \%$. Hence, about half of the templates can be disregarded in a search for SGRB progenitors, promising an increase in both speed and sensitivity.

This work was supported by STFC grant No. ST/L000342/1. We thank the LIGO-Virgo collaboration CBC and GRB groups for their stimulating environment, in particular Stephen Fairhurst, Raymond Frey, and Ian Harry for interesting discussions throughout the genesis of this work and for pushing the boundaries of this project further with their questions. We also thank Patricia Schmidt and Mark Hannam for many useful discussions about precessing binaries, and the anonymous referee for helpful comments.

\section{REFERENCES}

Aasi, J., Abadie, J., Abbott, B. P., et al. 2013, PhRvD, 88, 062001

Akmal, A., \& Pandharipande, V. R. 1997, PhRvC, 56, 2261

Akmal, A., Pandharipande, V. R., \& Ravenhall, D. G. 1998, PhRvC, 58, 1804

Alcock, C., \& Olinto, A. 1988, ARNPS, 38, 161

Berger, E. 2011, NewAR, 55, 1

Bildsten, L., \& Cutler, C. 1992, ApJ, 400, 175

Dal Canton, T., Nitz, A. H., Lundgren, A. P., Nielsen, A. B., \& Brown, D. A. 2014, arXiv:1405.6731

Damour, T., Iyer, B. R., \& Sathyaprakash, B. 2001, PhRvD, 63, 044023

Dominik, M., Berti, E., O'Shaughnessy, R., et al. 2014, arXiv:1405.7016

Farhi, E., \& Jaffe, R. L. 1984, PhRvD, 30, 2379

Foucart, F. 2012, PhRvD, 86, 124007

Foucart, F., Deaton, M. B., Duez, M. D., et al. 2013, PhRvD, 87, 084006

Harry, G. M., \& The LIGO Scientific Collaboration. 2010, CQGra, 27, 084006

Harry, I., Nitz, A., Brown, D. A., Lundgren, A., \& Ochsner, E. 2014, PhRvD, 89,024010

Kiziltan, B., Kottas, A., De Yoreo, M., \& Thorsett, S. E. 2013, arXiv:1309.6635 Kochanek, C. S. 1992, ApJ, 398, 234

Kyutoku, K., Shibata, M., \& Taniguchi, K. 2010, PhRvD, 82, 044049

Lackey, B. D., Kyutoku, K., Shibata, M., Brady, P. R., \& Friedman, J. L. 2012, PhRvD, 85, 044061

Lackey, B. D., Kyutoku, K., Shibata, M., Brady, P. R., \& Friedman, J. L. 2014, PhRvD, 89, 043009

Lattimer, J. M., \& Prakash, M. 2007, PhR, 442, 109

LIGO Scientific Collaboration, Virgo Collaboration, Aasi, J., et al. 2013, arXiv: 1304.0670

Maselli, A., \& Ferrari, V. 2014, PhRvD, 89, 064056

Nakar, E. 2007, PhR, 442, 166

Ohme, F., Nielsen, A. B., Keppel, D., \& Lundgren, A. 2013, PhRvD, 88, 042002 Pandharipande, V. R., \& Smith, R. A. 1975, NuPhA, 237, 507

Pannarale, F., Berti, E., Kyutoku, K., \& Shibata, M. 2013, PhRvD, 88, 084011

Pannarale, F., Rezzolla, L., Ohme, F., \& Read, J. S. 2011, PhRvD, 84, 104017

Pannarale, F., Tonita, A., \& Rezzolla, L. 2011, ApJ, 727, 95

Pekowsky, L., O’Shaughnessy, R., Healy, J., \& Shoemaker, D. 2013, PhRvD, 88, 024040

Rezzolla, L., Giacomazzo, B., Baiotti, L., et al. 2011, ApJL, 732, L6

Schmidt, P., Hannam, M., \& Husa, S. 2012, PhRvD, 86, 104063

Shoemaker, D. 2010, Advanced LIGO Anticipated Sensitivity Curves, https://dcc.ligo.org/cgi-bin/DocDB/ShowDocument?docid=2974

Singer, L. P., Price, L. R., Farr, B., Urban, A. L., \& Pankow, C. 2014, arXiv: 1404.5623

Somiya, K. 2012, CQGra, 29, 124007

Stone, N., Loeb, A., \& Berger, E. 2013, PhRvD, 87, 084053

Thorne, K. S. 1974, ApJ, 191, 507

Vallisneri, M. 2008, PhRvD, 77, 042001

Virgo Collaboration. 2009, Advanced Virgo Baseline Design, Virgo Technical Report VIR-0027A-09, https://tds.ego-gw.it/itf/tds/file.php?callFile=VIR0027A-09.pdf

Wade, L., Creighton, J. D. E., Ochsner, E., et al. 2014, PhRvD, 89, 103012

Wiringa, R. B., Fiks, V., \& Fabrocini, A. 1988, PhRvC, 38, 1010

Witten, E. 1984, PhRvD, 30, 272 